\title{
Construction of a recombinant lentivirus-mediated shRNA expression vector targeting the human PSMD10 gene and validation of RNAi efficiency in RPMI-8226 multiple myeloma cells
}

\author{
SIYUE DU ${ }^{1}$, WENJIAO QIN ${ }^{1}$, HAIYAN LENG $^{1}$, ZI CHEN $^{1}$ and TAO ZHANG ${ }^{2}$ \\ Departments of ${ }^{1}$ Hematology and ${ }^{2}$ Laboratory Medicine, Huashan Hospital Affiliated to Fudan University, \\ Jingan, Shanghai, P.R. China
}

Received November 9, 2016; Accepted June 9, 2017

DOI: $10.3892 /$ or.2017.5770

\begin{abstract}
Multiple myeloma (MM) is one of the most common malignant blood cancers. Previous studies have reported that proteasome 26S subunit non-ATPase 10 (PSMD10) is an oncoprotein with complex roles in hepatocellular carcinoma and other malignant tumors. Notably, research on the relationship between PSMD10 and tumorigenesis of MM has rarely been reported. The present study was designed to explore the possibility of PSMD10 as a therapeutic target in the treatment of MM, and the use of RNA interference (RNAi) to determine the function PSMD10. A recombinant lentivirus-mediated short hairpin RNA (shRNA) targeting human PSMD10 mRNA was constructed and used to decrease endogenous PSMD10 expression in the MM RPMI-8226 cell line in vitro. Expression of the PSMD10-targeting shRNA in RPMI-8226 cells transduced with the recombinant vector could be tracked by observing the expression of green fluorescent protein after infection. A transient transgenic RPMI-8226 cell line was generated by transducing cells with the packaged viral particles. Western blot analysis indicated that the protein levels of PSMD10 in the PSMD10-shRNA MM cells were significantly lower than those in the cells transduced with the negative control shRNA. Notably, RT-qPCR analysis did not reveal a marked change in the PSMD10 mRNA level; thus, the knockdown effect of the PSMD10-shRNA may occur during translation. Furthermore,
\end{abstract}

Correspondence to: Dr Tao Zhang, Department of Laboratory Medicine, Huashan Hospital Affiliated to Fudan University, 12 Wulumuqi Middle Road, Jingan, Shanghai, P.R. China E-mail: shmuzt@126.com

Professor Zi Chen, Department of Hematology, Huashan Hospital Affiliated to Fudan University, 12 Wulumuqi Middle Road, Jingan, Shanghai, P.R. China

E-mail: drchenzi@163.com

Key words: PSMD10, lentiviral vector, short hairpin RNA, RNA interference, RPMI-8226 cells, multiple myeloma apoptosis of MM cells was increased by silencing PSMD10 expression. Overall, the results demonstrated that the lentivirus-mediated shRNA vector-based RNAi expression system is an efficient method to silence PSMD10 gene expression in the MM RPMI-8226 cell line. It may provide a basis to study the role of PSMD10 in tumor cells, and may be a reliable gene therapy strategy in the clinic.

\section{Introduction}

Multiple myeloma (MM) is a malignancy of the bone marrow characterized by the clonal proliferation of antibody-secreting plasma cells (1). The incidence of MM has exceeded that of acute leukemia, ranking as the second most common of all hematological malignancies. Worldwide, $>80,000$ new cases of MM are reported each year, representing $10 \%$ of all hematological malignancies and $\sim 1 \%$ of all new cancer cases (2-3). Abnormal proliferation of plasma cells produces monoclonal immunoglobulins (paraprotein, M-component, M-protein) and gives rise to symptoms and conditions including hypercalcemia, renal impairment, pathological bone fractures, hyperviscosity and bone marrow deficiency by suppressing the function of normal immunoglobulins.

The etiology of MM is not fully understood. Radiation, chemicals, certain viruses, weakened immunity and chromosomal abnormalities are thought to be involved in MM etiology (4). An association between proteasome 26S subunit non-ATPase 10 (PSMD10) and the tumorigenesis of MM has not been widely investigated; however, aberrant expression of PSMD10 has been reported to have an important function in various other malignancies, including gastric cancer, intrahepatic cholangiocarcinoma, testicular germ cell tumors and hepatocellular carcinomas (5-9). In a preliminary study, a Protein Turnover Assay (ProTA) was developed; the assay insures strict co-translational expression of duplicate monomeric fluorescent proteins that possess the natural $\mathrm{N}$-terminal residues of 15,000 human proteins, which therefore allows precise snapshot profiling of global protein stability at the system level (10). Subsequently, ProTA was applied to profile changes in human protein degradation induced by bortezomib (BTZ), aiming to elucidate the potential molecular basis of the effects 
of BTZ and tumor drug resistance. Additionally, it was ascertained that BTZ stabilized proteasome 26S subunit ATPase 1 (PSMC1) and the proteasome assembly factor PSMD10 (10). PSMD10, also known as gankyrin or p28 (GANK), is one of the non-ATPase subunits that is a regulatory component of the human 26S proteasome. PSMD10, the mammalian homolog of yeast proteasome assembly factor NAS6 (11), controls the balance between the cell cycle and apoptosis by degrading RB1 and TP53 (12,13), which have important effects on the pathogenesis of cancer. Dysregulation of PSMD10 expression leads to cancer progression, and has crucial functions in the processes of cell survival, migration and proliferation (14-16). Previous studies reported that the direct downregulation of PSMD10 expression in MM cells induced an increase of P53 mRNA levels and subsequent upregulation of cyclin-dependent kinase inhibitor 1A (CDKN1A; also known as Cip1/p21Waf1) and BAX transcripts, which are direct transcriptional targets of P53 (17). PSMD10 acts as an oncoprotein and is involved in the negative regulation of the tumor suppressors RB1 and P53 (13). The imbalance of P53 in myeloma cells may play an important role in myeloma pathogenesis. Upon BTZ-induced proteasome inhibition, myeloma cells appear to acutely stabilize the proteasome assembly factor, PSMD10, which ultimately facilitates the assembly of proteasome subunits into functional proteasomes (10). Thus, we hypothesized that this may functionally abrogate BTZ-induced proteasome inhibition and help cells survive proteotoxic stress, which may provide an escape route for cells, resulting in tumorigenesis, progression and drug resistance during BTZ-based chemotherapy. This hypothesis may be further examined by designing RNA interference (RNAi) tools that specifically target the pro-survival functions of PSMD10 in tumorigenic cells on the basis of the tissue distributions and the differential functions of the various PSMD10 splice variants.

RNAi is a sequence-specific gene silencing process that occurs at the mRNA level. In mammalian cells, short dsRNAs ( $<30$ bp) knockdown the expression of specific mRNAs via base pairing (18). Based on this physiological process, silencing of gene expression by RNAi is currently used as a standard tool in cultured mammalian cells and has had a considerable impact on research into the function of human genes and gene therapy (19). Although chemically synthetic siRNAs can be introduced into cultured cells and induce a transient knockdown effect of target mRNAs, this does not always result in high efficiency of RNAi delivery and stable transcript knockdown. Alternatively, in mammalian cells, expression vectors driven by RNA polymerase III enable the permanent production of small dsRNAs $(20,21)$. In addition, a lentiviral system can be employed to reversibly induce gene silencing in a spatially and temporally restricted manner.

The relationship between PSMD10 and MM, and the function of PSMD10 in MM cells, have not been clearly established yet. In the present study, a recombinant lentivirus-mediated short hairpin RNA (shRNA) vector targeting human PSMD10 gene was designed. The vector-produced RNAs were processed by Dicer in a similar manner to siRNAs. The recombinant lentivirus vector mediating the RNAi-targeting of the PSMD10 gene was then used to examine the effect of PSMD10 knockdown on RPMI-8226 MM cells in vitro.

\section{Materials and methods}

Cell culture. RPMI-8226 MM and 293T cells were obtained from the Cell Institute of the Chinese Academy of Sciences (Shanghai, China). RPMI-8226 cells were cultured in RPMI-1640 culture-medium and 293T cells in Dulbecco's modified Eagle's medium (DMEM), supplemented with $10 \%$ fetal bovine serum (FBS; Gibco), $100 \mathrm{U} / \mathrm{ml}$ penicillin and $100 \mathrm{mg} / \mathrm{ml}$ streptomycin (all from Invitrogen, Carlsbad, CA, USA). Cells were incubated at $37^{\circ} \mathrm{C}$ with $5 \% \mathrm{CO}_{2}$, the medium was refreshed every 24 or $48 \mathrm{~h}$, and $293 \mathrm{~T}$ cells were passaged using trypsin solution when confluent monolayers were reached.

Design of human PSMD10-shRNA. The mRNA sequence of human PSMD10 (GenBank; NM_002814.3) was inputted into the Invitrogen online RNAi Designer to design siRNAs. We selected three optimal siRNAs that targeted different sequences, as not every siRNA sequence is equally effective when incorporated into an shRNA (22). A control scrambled shRNA sequence was specifically designed. All four sequences were aligned using the GenBank BLAST program, no homologous sequences matched other than the PSMD10 gene. In order to obtain the dsRNA configuration required for shRNA formation from single-stranded RNA, a sense siRNA sequence was linked to its complementary antisense sequence via a 12-bp loop region, and combined with two supplementary $\mathrm{T}$ nucleotides flanked by restriction enzyme recognition sequences and protective bases (23). Two complementary single-stranded DNA oligonucleotides of the four shRNAs were chemically synthesized by the Laboratory of Pharmacology, Second Military Medical University (Shanghai, China). These oligonucleotides were annealed to produce double-stranded oligonucleotides (Table I).

Construction of pSIH-PSMD10-shRNA vectors. The annealed PSMD10-shRNA oligonucleotides were cloned into linearized pSIH1-H1-copGFP shRNA empty vectors (catalog no. SI501A-1; System Biosciences) using the following steps: pSIH1-H1-GFP shRNA vectors and double-stranded shRNA oligonucleotides were digested with both BamHI and EcoRI; and the large fragment of the vector and the digested shRNA fragment were then ligated using T4 DNA ligase according to the manufacturer's instructions. The ligated products were transformed into competent DH5 $\alpha$ cells (catalog no. D9057; Takara, Shiga, Japan) using the heat shock method. The transformed cells were grown on an LB-agar plate containing ampicillin at $37^{\circ} \mathrm{C}$ overnight.

Identification of double digestion and DNA sequence. To ensure that the shRNAs were inserted into the vectors, positive clones were collected and identified by PCR amplification. PCR products $(5 \mu \mathrm{l})$ were separated using $2 \%$ agarose gel electrophoresis and the lengths of the amplified bands were examined. The primer sequences used for PCR identification were as follows: forward, 5'-ATAT TTGC ATGT CGCT ATGT G-3' and reverse, 5'-CAGG CTAG ATCT GGTC TAAC CA-3'. Positive clones were cultured overnight at $37^{\circ} \mathrm{C}$ in $3 \mathrm{ml} \mathrm{LB}$ broth plus $100 \mu \mathrm{g} / \mathrm{ml}$ ampicillin with vigorous agitation at $200 \mathrm{rpm}$. The plasmid DNA was prepared using Mini Plasmid Purification 
Table I. Sequences of 4 designed PSMD10-shRNA.

PSMD10-shRNA

name
Sequences
shRNA1-f

shRNA1-r

shRNA2-f

shRNA2-r

shRNA3-f

shRNA3-r

NC-f

NC-r
5'-GATCCGCCGATAAATCCCTGGCTACTTCCTGTCAGATAGCCAGGGATTTATCGGCTTTTTG-3 5'-AATTCAAAAAGCCGATAAATCCCTGGCTATCTGACAGGAAGTAGCCAGGGATTTATCGGCC-3' 5'-GATCCGGCTGTACTCCCTTACATTCTTCCTGTCAGAAATGTAAGGGAGTACAGCCTTTTTG-3' 5'-AATTCAAAAAGGCTGTACTCCCTTACATTTCTGACAGGAAGAATGTAAGGGAGTACAGCCC-3' 5'-GATCCGGCATGAGATCGCTGTCATCTTCCTGTCAGAATGACAGCGATCTCATGCCTTTTTG-3' 5'-AATTCAAAAAGGCATGAGATCGCTGTCATTCTGACAGGAAGATGACAGCGATCTCATGCCC-3' 5'-GATCCGAAGCCAGATCCAGCTTCCCTTCCTGTCAGAGGAAGCTGGATCTGGCTTCTTTTTG-3' 5'-AATTCAAAAAGAAGCCAGATCCAGCTTCCTCTGACAGGAAGGGAAGCTGGATCTGGCTTCC-3'

f, forward; r, reverse; shRNA, short hairpin RNA.

Table II. Primers of PSMD10 and $\beta$-actin.

\begin{tabular}{lcc}
\hline Name & \multicolumn{1}{c}{ Primer } & Product length (bp) \\
\hline Human PSMD10 & & 187 \\
Sense & 5'-CTGGCCGGGATGAGATTGTAAAAG-3' & \\
Antisense & 5'-CGGTGCATTGCTGTAGCCTCATAA-3' & \\
Human $\beta$-actin & & 219 \\
Sense & 5'-CCCAAGGCCAACCGCGAGAAGATG-3' \\
Antisense & 5'-GTCCCGGCCAGCCAGGTCCAGA-3' & \\
\hline
\end{tabular}

kit Ver 2.0 (catalog no. SK1191; Shanghai ShengGong Biotech, Shanghai, China) according to the manufacturer's instructions. Extracted recombinant plasmids were used for DNA sequencing to identify the inserted PSMD10-shRNA fragments. The pSIH1-PSMD10-shRNA sequencing forward primer was: 5'-GCAGAGCTCGTTTAGTGAACC-3'.

Effective siRNA sequence screening and verification. The recombinant expression vector was verified by sequencing extracted plasmid DNA. Endotoxins were removed using a plasmid mini kit (catalog no. 12145; Qiagen, Inc., Hilden, Germany) according to the manufacturer's instructions. The purity and concentration of plasmid DNA were determined by UV spectrophotometer after observing the integrity of plasmid DNA on agarose gel electrophoresis.

When $293 \mathrm{~T}$ cells cultured in a $10-\mathrm{cm}$ cell culture dish had reached $70 \%$ confluence, a cell suspension was prepared using trypsin. The cells were then seeded into a 6-well plate in $2 \mathrm{ml}$ complete DMEM (catalog no. 11995073; Invitrogen) plus $10 \%$ FBS, and cultured at $37^{\circ} \mathrm{C}$ in $5 \% \mathrm{CO}_{2}$ overnight. After $24 \mathrm{~h}$, adherent cells reached $\sim 80 \%$ confluence and were in a healthy condition with spindle-like shape. For transfection, $4 \mu \mathrm{g}$ plasmid DNA diluted in $250 \mu \mathrm{l}$ Opti-MEM (catalog no. 11058021) and $10 \mu \mathrm{l}$ Lipofectamine 2000 (catalog no. 1668027) (both from Invitrogen) diluted in $250 \mu \mathrm{l}$ Opti-MEM were used/well according to this protocol. Transfected cells were grouped as follows: 293T, 293T + pshRNA vector, $293 \mathrm{~T}+$ pshRNA1-PSMD10, 293T + pshRNA2-PSMD10,
$293 \mathrm{~T}+$ pshRNA3-PSMD10, 293T + pshRNA-NC. After transfection for $48 \mathrm{~h}$, fluorescence microscopy indicated that the transfection efficiency was $\sim 90 \%$.

Total RNA was extracted from the transfected cells using TRIzol (catalog no. 15596-018; Invitrogen). Extracted RNA was treated with DNase I to remove any DNA contamination, and single-stranded cDNA was prepared from RNA using Reverse Transcriptase M-MLV (catalog no. D2640A; Takara). Quantitative PCR (qPCR) primers were designed using Primer Premier 5.0 and oligo 7.0. A reverse transcription-qPCR reaction was performed to screen the most effective recombinant plasmids. qPCR was performed using Takara SYBR Premix Ex Taq (catalog no. DRR041A; Takara). A standard PCR program was used as follows: $95^{\circ} \mathrm{C}$ for $3 \mathrm{~min} ; 40$ cycles of $95^{\circ} \mathrm{C}$ for $10 \mathrm{sec}$ and $60^{\circ} \mathrm{C}$ for $20 \mathrm{sec}$; followed by $95^{\circ} \mathrm{C}$ for $10 \mathrm{sec}$ and $72^{\circ} \mathrm{C}$ for $20 \mathrm{sec}$. A melting curve analysis was performed to verify the identities of PCR products. Each sample was tested three times to obtain an average. Relative expression levels of the PSMD10 gene were normalized to $\beta$-actin expression levels by calculating the $\mathrm{Cq}$ value using Thermal Cycler DICE Real-Time System analysis software. Primer sequences for the amplification of PSMD10 and $\beta$-actin are listed in Table II.

Lv-shRNA2-PSMD10 recombinant virus packaging and production. pPACKTM Lentivector Packaging System was used to perform the packaging and production of lentiviral particles. At $24 \mathrm{~h}$ before transfection, 293TN packaging cells were seeded in a $10-\mathrm{cm}$ cell culture dish to achieve 
$\sim 80 \%$ confluence for the transfection experiment. Per $10-\mathrm{cm}$ culture dish, $4 \mu \mathrm{l}$ of the most effective recombinant plasmid pshRNA2-PSMD10 (500 $\mathrm{ng} / \mu \mathrm{l})$ and $20 \mu 1$ Lentivirus Package plasmid mix (500 ng/ $\mu$ l) (catalog no. LV500A-1; System Biosciences) were co-transfected into cells using $40 \mu \mathrm{l}$ transfection reagent according to the manufacturer's instructions. At $24 \mathrm{~h}$ after transfection, the medium was refreshed using freshly prepared DMEM plus $2 \%$ FBS. At $48 \mathrm{~h}$ after transfection, the cells were adherent and $\sim 90 \%$ confluent with normal growth conditions and a flat shape. Viral particle-containing supernatants were harvested by removing the medium from the cells and transferred to a $15-\mathrm{ml}$ sterile, capped, conical tube. Non-adherent cells and debris were pelleted by centrifugation at $5,000 \mathrm{x}$ g at $4^{\circ} \mathrm{C}$ for $5 \mathrm{~min}$. Each viral titer was calculated as $\times 10^{4}$ by counting the number of $\mathrm{GFP}^{+} 293 \mathrm{~T}$ cells by the gradient dilution method 2 days after transduction with serial dilutions of the viral stocks. The virus particle concentration was adjusted to $1 \times 10^{4} \mathrm{ifu} / \mu 1$ using $\mathrm{dPBS}$ $(\mathrm{pH}=7.8)$ with $1 \mathrm{ml}$ of each tube packaged and stored at $-80^{\circ} \mathrm{C}$.

Recombinant lentiviral infection of target cells. The optimum MOI value of the lentivirus infection of RPMI-8226 cells was determined according to the results of preliminary experiments. An MOI of 20 was used. RPMI- 8226 cells were seeded in a 6-well plate and cultured in RPMI-1640 plus 10\% FBS. Infected cells were grouped as follows: RPMI-8226 cells, RPMI-8226 cells + Lv-NC, RPMI-8226 + Lv-shRNA2PSMD10. The virus solution was added to the cells at the optimum MOI of 20. Fluorescence was enhanced and the number of infected cells ( 90\%) was increased at $96 \mathrm{~h}$ postinfection compared with that at $72 \mathrm{~h}$. Some cells were collected for RNA and protein extraction to evaluate RNAi efficiency, and the remaining cells were stored in liquid nitrogen for further experiments.

Detection of gene and protein levels in target cells. Total RNA was extracted from the MM RPMI-8226 cells using experimental methods and reagents as previously described. MM cells were lysed in $1 \mathrm{ml}$ M-PER Mammalian Protein Extraction Reagent (catalog no. 78501; Pierce, Rockford, IL, USA) plus protease inhibitor, then incubated on ice for $40 \mathrm{~min}$. The lysates were cleared by centrifugation and the protein concentration was determined using the BCA method. Proteins were separated by SDS-PAGE (4\% stacking gel, $10 \%$ separating gel) and transferred to polyvinylidene difluoride (PVDF) membranes (catalog no. IPVH00010; Millipore, Billerica, MA, USA). The membranes were blocked with 5\% skimmed milk in Tris-buffered saline/Tween-20 (TBST) buffer, then probed with anti-PSMD10 (catalog no. ab154676; Abcam, Cambridge, MA, USA) and anti- $\beta$-actin (catalog no. sc-81178; Santa Cruz Biotechnology, Inc., Santa Cruz, CA, USA) antibodies at room temperature, followed by incubation with horseradish peroxidase-conjugated anti-rabbit (for PSMD10; catalog no. 7074) and anti-mouse (for $\beta$-actin; catalog no. 7076) secondary antibodies. After several washes, the membranes were developed with an enhanced chemiluminescence system (catalog no. 32106; Pierce ECL Western Blotting Substrate) and exposed to Kodak BioMax light film. $\beta$-actin protein levels were used as a control to verify equal protein loading.

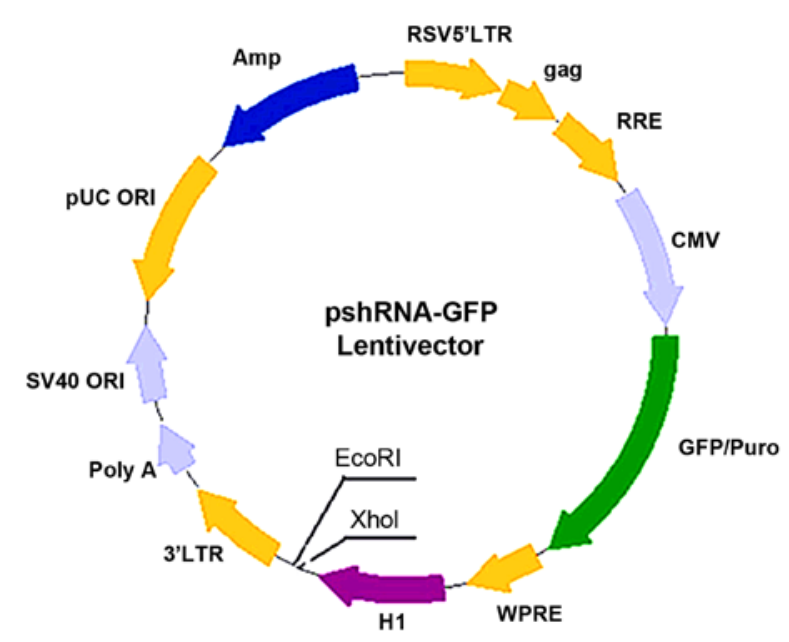

Figure 1. Diagram of lentiviral vectors expressing PSMD10 with GFP. cPPT, central polypurine tract; LTR long terminal repeat; WPRE, woodchuck hepatitis post-transcriptional regulatory element; PSMD10, target gene; GFP, green fluorescence protein.
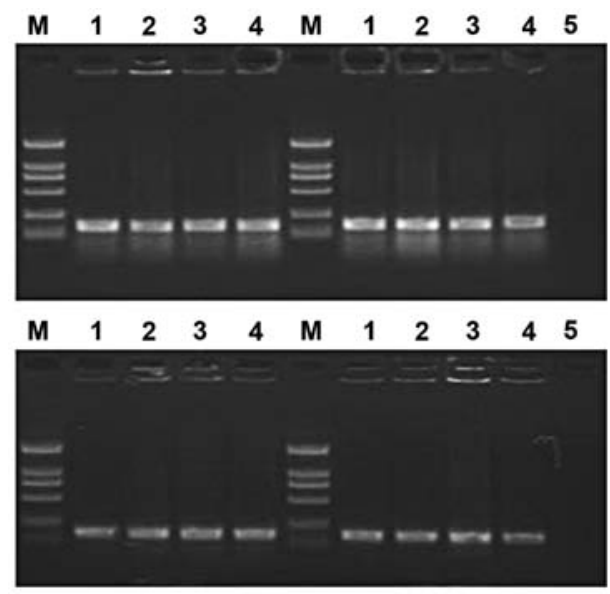

Figure 2. Identification of positive clones on $2 \%$ agarose gel, $110 \mathrm{v}, 10 \mathrm{~min}$ on constant voltage, $213 \mathrm{bp}$ of PCR product length of positive clones (insertion sequence $61 \mathrm{bp}+$ vector multiple cloning sites $152 \mathrm{bp}$ ) was seen in the p1-4 groups. M, DL 2,000 DNA Marker (D501; Takara); lane 1-4, all clone colony of 4 groups were PCR products of template; lane 5, negative control of PCR reaction system. All detected colonies were positive, and no bands were seen in the p5 (NC), showing that PCR system was not contaminated.

Detection of apoptosis by flow cytometry and fluorescence microscopy. After transduction with the lentivirus, MM cell lines were seeded in 6-well plates at a density of $1 \times 10^{5}$ cells/well. After $96 \mathrm{~h}$ post-infection, MM cells were harvested and washed in phosphate-buffered saline (PBS). A cell suspension with a density of $1 \times 10^{6}$ cells $/ \mathrm{ml}$ was prepared for each assay. Cells were simultaneously stained with phycoerythrin (PE)-labeled Annexin $\mathrm{V}$ and with 7-aminoactinmycin D (7-AAD) to discriminate viable cells (Annexin $/ 7-\mathrm{AAD}^{-}$) from early apoptotic cells (Annexin ${ }^{+} / 7-\mathrm{AAD}^{-}$), and late apoptotic and necrotic cells (Annexin ${ }^{+} / 7-\mathrm{AAD}^{+}$). Flow cytometric analysis was performed to detect Annexin $\mathrm{V}$ and 7-AAD staining. All experiments were performed in triplicate.

Statistical analysis. SPSS version 21.0 was used for statistical analysis. Values presented are representative of triplicate 


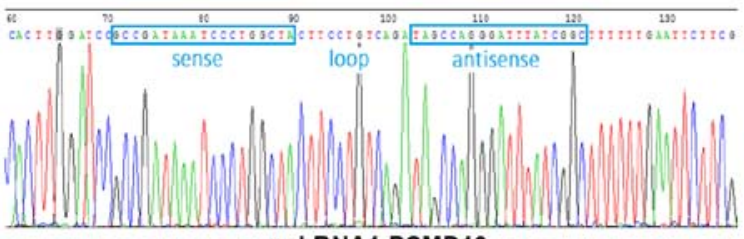

pShRNA1-PSMD10

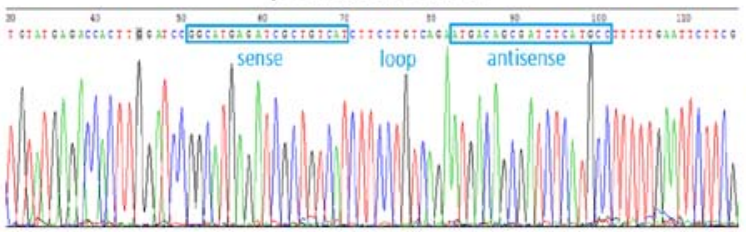

pShRNA3-PSMD10

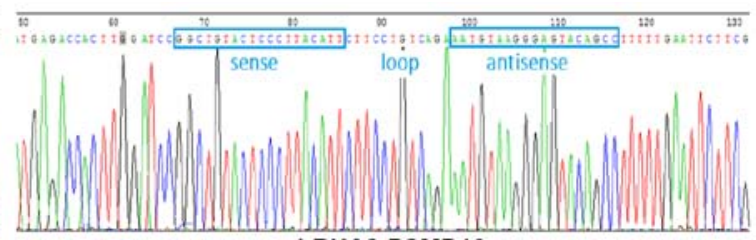

pshRNA2-PSMD10

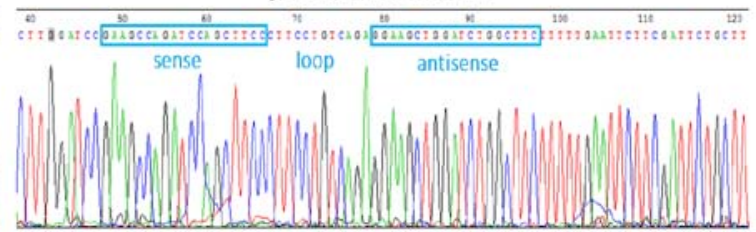

pshRNA-NC

Figure 3. As is shown in the image above, the results of DNA sequencing were completely consistent with the designed sequence.

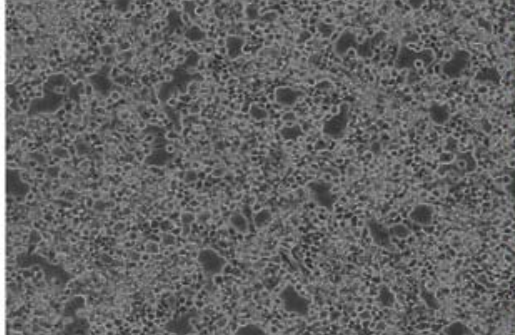

po

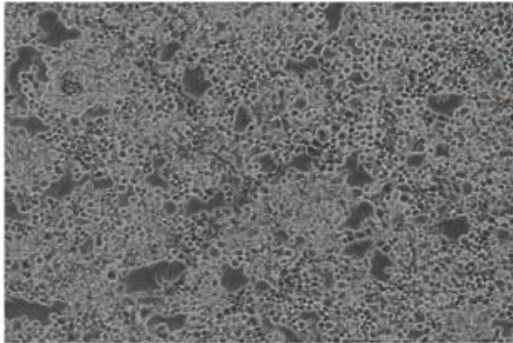

p1

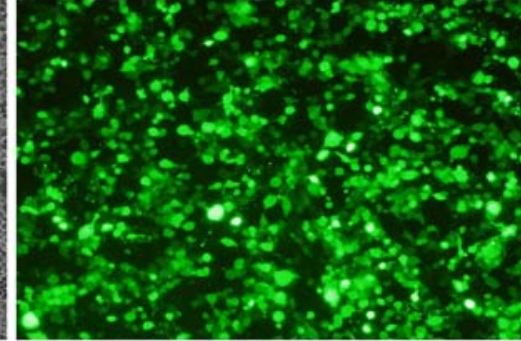

p0 after $48 \mathrm{~h}$

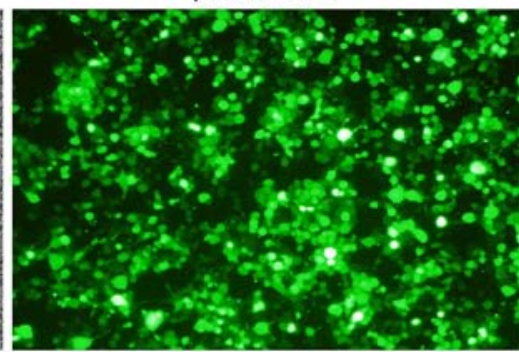

p1 after $48 \mathrm{~h}$

Figure 4. The images of 293 T cell transfection (magnification, x100) are shown: pshRNA-vector plasmid transfection (p0) and pshRNA2-PSMD10 recombinant expression vector plasmid transfection $(\mathrm{p} 1)$.

determinations in no less than three experiments. Data are expressed as the mean \pm standard deviation and compared using a two-sided Student's t-test. $\mathrm{P}<0.05$ was considered to indicate a statistically significant difference.

\section{Results}

Sequencing profile of the recombinant PSMD10-shRNA expression vectors. A schematic diagram of the construction of the pSIH1-H1-shRNA expression vector to silence PSMD10 gene expression is presented in Fig. 1. Recombinant plasmids were digested with $B a m \mathrm{HI}$ and EcoRI, and fragments were identified on $2 \%$ agarose gels (Fig. 2). DNA sequencing results provided further verification of the presence of the recombinant plasmids, indicating that the correct sequences were carried by all the shRNA expression plasmids (Fig. 3).

Detection of $293 T$ cell transfection efficiency and RT-qPCR. After transfection for $48 \mathrm{~h}$, a fluorescence microscope was used to observe the transfection efficiency of $293 \mathrm{~T}$ cells transfected with the pSIH1-H1-shRNA expression

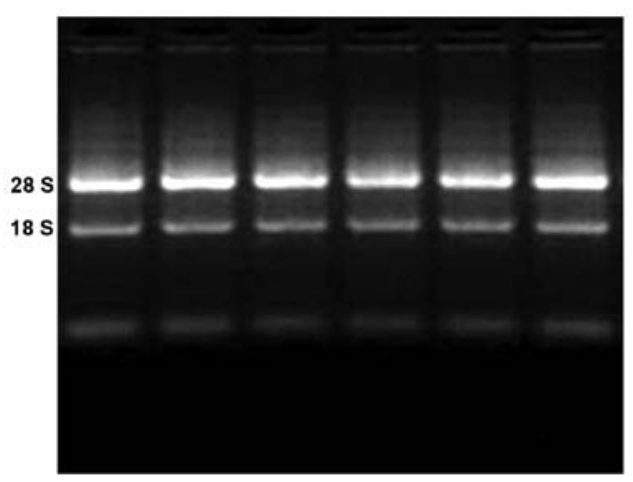

Figure 5. The detection results of the RNA purity revealed that the RNA ratio of A260/A280 nm was between 1.8 and 2.0. Two bands of $18 \mathrm{~S}$ and 28S RNA were clearly visible, indicating a higher purity of RNA without obvious degradation and genomic contamination.

vector; the transfection efficiency was $~ 90 \%$ (Fig. 4). RNA electrophoresis was performed to demonstrate the integrity of extracted RNA (Fig. 5). RT-qPCR was performed to 


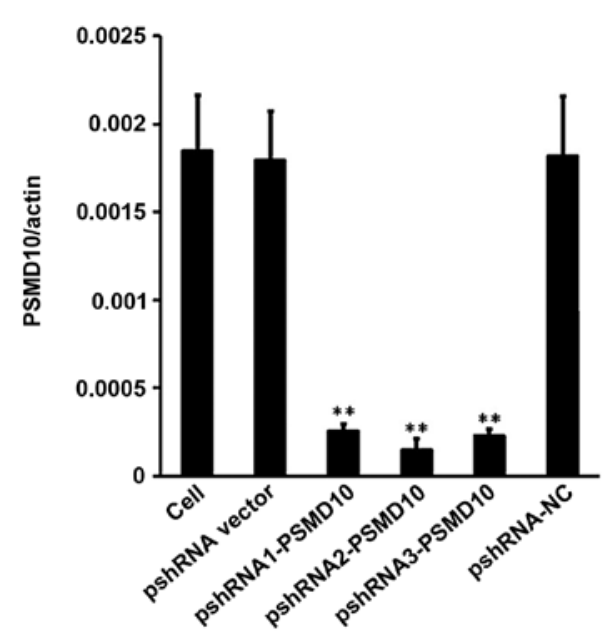

Figure 6. Relative expression levels of PSMD10 gene were normalized to $\beta$-actin expression levels by calculating the $\mathrm{Ct}$ value. As is shown in the figure above, the levels of PSMD10/actin were significantly lower in the pshRNA-PSMD10 (1-3) groups than in the cell control group $\left({ }^{* *} \mathrm{P}<0.01\right.$, t-test, respectively), particularly in the pshRNA2-PSMD10 group $(\mathrm{P}=0.0007)$.

analyze the relative expression levels of PSMD10 normalized to $\beta$-actin by calculating the $\mathrm{Ct}$ value using Thermal Cycler DICE Real-Time System analysis software (Fig. 6).

Infection of RPMI-8226 cells with Lv-shRNA2-PSMD10. RPMI-8226 cells infected with Lv-shRNA2-PSMD10 were observed to exhibit green fluorescence under a fluorescence microscope (Fig. 7).

Detection of PSMD10 mRNA levels after infection. The mRNA levels of PSMD10 were determined by RT-PCR (Fig. 8A) and RT-qPCR (Fig. 8B). Surprisingly, there was no significant difference in expression levels of PSMD10 mRNA between cells infected with Lv-shRNA2-PSMD10 and those infected with Lv-NC.

Silencing of PSMD10 protein expression after infection. Protein levels of PSMD10 gene expression were determined by western blotting. As demonstrated in Fig. 9A, compared with the non-infected cells and the Lv-NC group, the PSMD10 protein levels in the Lv-shRNA2-PSMD10 group were significantly decreased. Furthermore, there was a significant decrease in the protein expression of PSMD10 as demonstrated by densitometric analysis $(\mathrm{P}<0.01$; Fig. 9B).

Apoptosis is increased in MM cells by knockdown of endogenous PSMD10. To determine whether apoptosis was increased in MM RPMI-8226 cells by silencing PSMD10 gene expression, Annexin V-PE and 7-AAD staining was performed. Flow cytometric analysis was used to evaluate the number of apoptotic cells (Fig. 10). Silencing of PSMD10 increased the percentage of apoptotic cells (quadrant $2+$ quadrant 4 ) in the LV-shRNA2-PSMD10 group compared with the NC groups (Fig. 11).

\section{Discussion}

Using conventional treatments, MM remains an essentially incurable disease. The overall median survival time after an MM diagnosis is 5-6 years (24), yet disease outcomes are highly influenced by the characteristics of the cancer (for example, high-risk cytogenetics) or the patients (for example, age). Treatment of MM remains highly individualized, with multiple factors used to determine the most appropriate course of therapy. In younger patients, progression-free survival (PFS) and overall survival (OS) have been greatly improved by treatment with autologous stem cell transplantation $(25,26)$. Unfortunately, the decision-making process of treatment
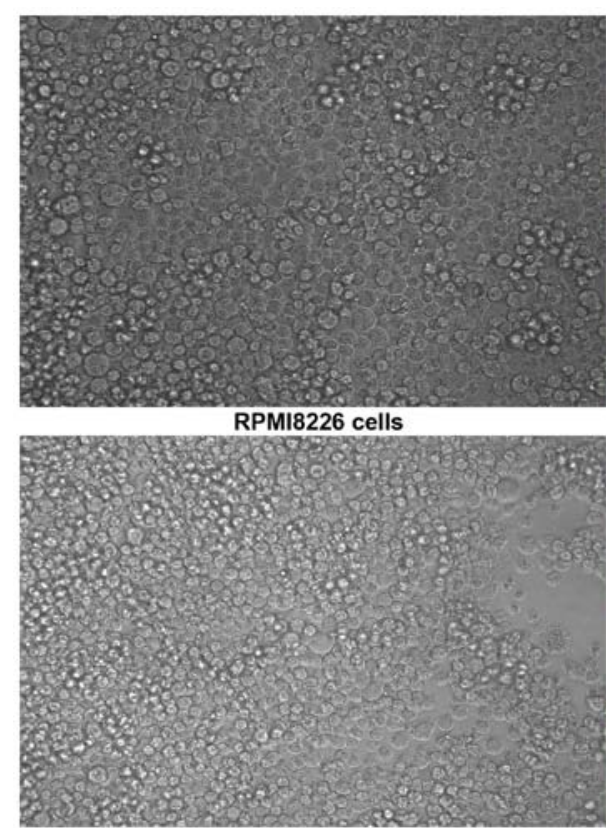

RPMI8226 cells

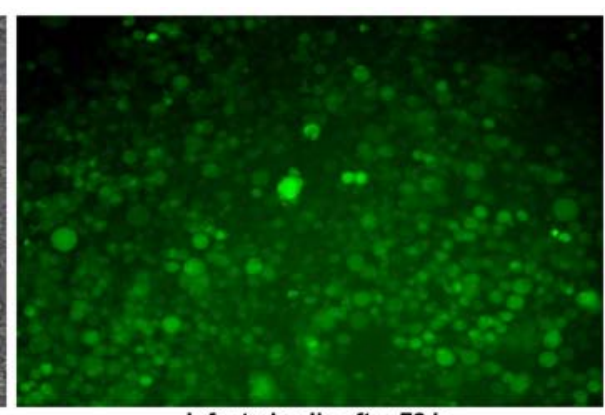

Infected cells after $72 \mathrm{~h}$

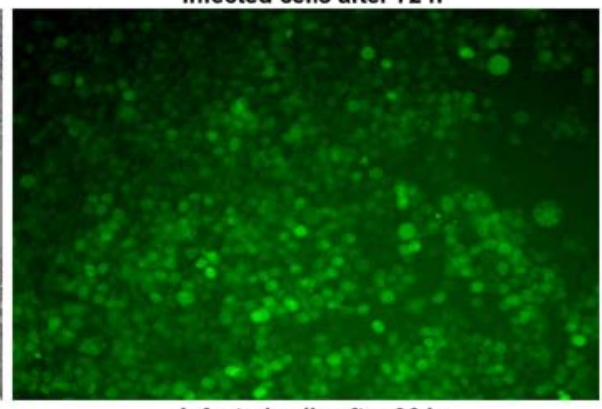

Infected cells after $96 \mathrm{~h}$

Figure 7. Infected RPMI-8226 cells (Lv-shRNA2-PSMD10) exhibited green fluorescence (magnification, x120) at 72 and $96 \mathrm{~h}$ respectively, and fluorescence was particularly enhanced at the latter time. $\mathrm{MOI}=20$. 


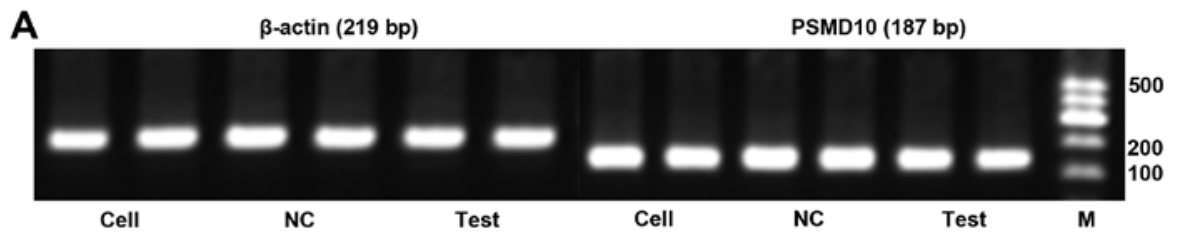

B

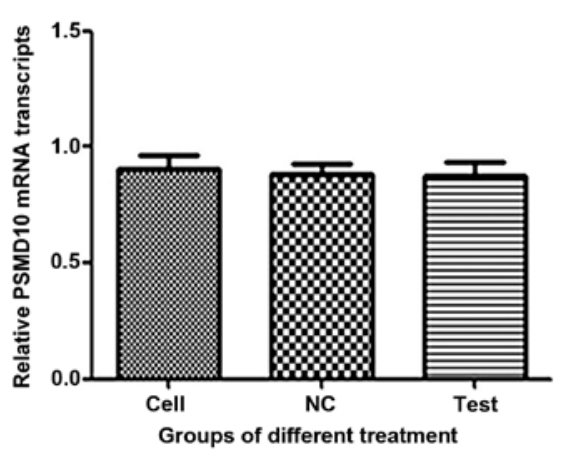

Figure 8. (A) PSMD10 mRNA is determined by reverse transcription PCR. The PCR products were separated on a $2 \%$ agarose gel marker, $1 \mathrm{~kb}$. We observed no significant change in the band levels of the test group (Lv-shRNA2-PSMD10 group) compared with the cell group. (B) Real-time quantitative PCR showed that there was no significant change among the cell, $\mathrm{NC}$ and test groups $(\mathrm{P}>0.05)$.
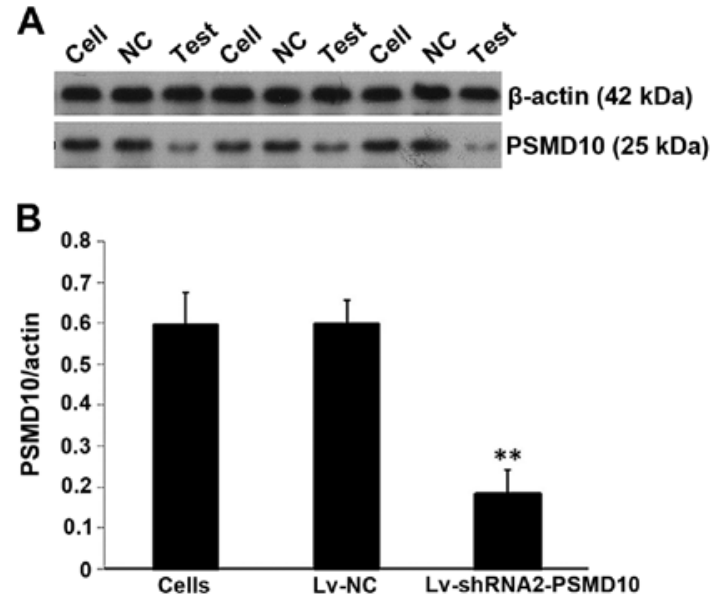

Figure 9. (A) The protein levels of PSMD10 were greatly decreased in the test group compared with both cells and NC groups by performing densitometric analysis. Each sample was performed in triplicate. (B) By further statistical analysis, we observed that there was an obvious change in the test group (Lv-shRNA2-PSMD10) compared with the other 2 groups $\left({ }^{* *} \mathrm{P}=0.001\right.$, respectively).

choices for myeloma patients at each phase of the disease has become complex; this is in large part due to the deeper understanding of plasma cell biology, resulting in more therapeutic options at each stage. MM is an incurable plasma cell malignancy characterized by initial responsiveness to treatment, followed by the appearance of increasingly refractory disease and ultimately, death due to infection, renal failure and cytopenia (27). Thus, the development of new therapeutic strategies for MM remains important.

In recent years, the proteasome inhibitors (PIs) BTZ and carfilzomib have emerged as frontline treatments for relapsed and refractory MM; however, resistance to these drugs occurs through poorly defined mechanisms. Numerous studies have identified different acquired-resistance models, such as $\beta 5$ proteasome subunit mutations, and stabilization of tumor suppressors and pro-apoptosis proteins. In MM cells, sensitivity to proteasome inhibition arises since these malignant proliferative B cells are highly reliant on protein synthesis and turnover, and thus, highly dependent on the ubiquitin proteasome system for the processing of defective proteins (28-30). Various E1, E2 and E3 ligases tag targeted proteins with multiple ubiquitin residues, and the tagged proteins are subsequently degraded by the $26 \mathrm{~S}$ proteasome. A $19 \mathrm{~S}$ lid complex and a $20 \mathrm{~S}$ core complex form the $26 \mathrm{~S}$ proteasome. The $20 \mathrm{~S}$ core carries out the cleavage of unfolded proteins. Upon immune stimulation, for instance, by interferon- $\gamma$, the $20 \mathrm{~S}$ constitutive catalytic subunits, $\beta 1$ (PSMB6, caspase-like activity), $\beta 2$ (PSMB7, trypsin-like activity), and $\beta 5$ (PSMB5, chymotrypsin-like activity), are replaced by their immune cognate forms; $\beta 1 \mathrm{i}$ (PSMB9/LMP2), $\beta 2 \mathrm{i}$ (PSMB10/MECL-1) and $\beta 5 \mathrm{i}$ (PSMB8/LMP7) $(31,32)$. BTZ is a reversible inhibitor that targets primarily the $\beta 5$-subunit and also targets the caspase-like activity of the $\beta 1$ proteasome subunit to a lesser extent. At higher concentrations, BTZ inhibits the trypsin-like proteolytic activity facilitated by the $\beta 2$ proteasome subunits.

Previous research indicated that the molecular mechanisms involved in acquired BTZ resistance may be associated with the upregulation of $\beta 5$ subunits, the point mutation of $\beta 5$ genes and downregulation of $\beta 5$ i subunits (33). Some data suggested that $\beta 5$ subunits may be the key regulatory factors linked to the PI resistance of tumor cells. However, mutations of $\beta 5$ subunits may not be uniquely responsible for the resistance of tumor cells to PI drugs. An aberration in the PSMB8 gene was demonstrated to breakdown the tertiary structure of $\beta 5 \mathrm{i}$ and decrease chymotrypsin-like activity but did not affect $\beta 5$ i protein expression (34). Defects in immunoproteasome assembly in PSMB8 mutants can be expected, as $\beta 5 \mathrm{i}$ is required for the maturation of immunoproteasomes by processing and incorporation of the $\beta 1 \mathrm{i}$ and $\beta 2 \mathrm{i}$ subunits (35). Certain studies have reported that there may be an association between 

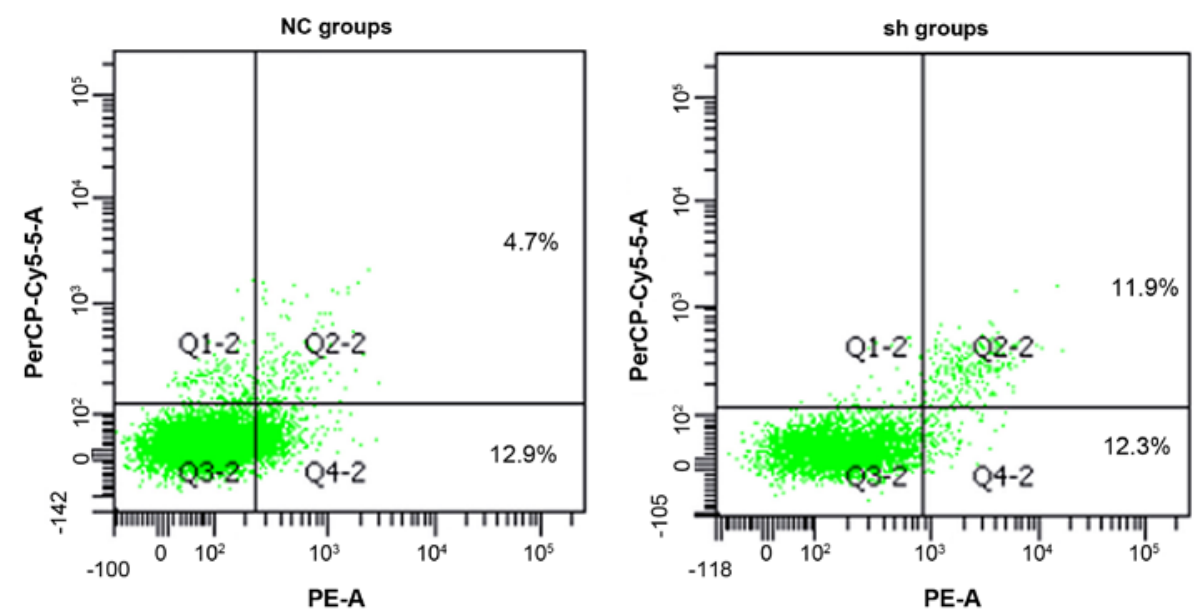

Figure 10. Using Annexin V-PE (X, PE-A) and 7-aminoactinmycin D (7-AAD; FL3 channel, Y, PerCP-Cy5.5) staining, normal living cells were Annexin V-PE and 7-AAD negative (lower left corner, Q3-2). Early apoptotic cells were Annexin V-PE positive only (lower right corner, Q4-2). Advanced necrotic and late apoptotic cells were both Annexin V-PE and 7-AAD positive (upper right corner, Q2-2). The upper left corner (Q1-2) is a detection error scope.

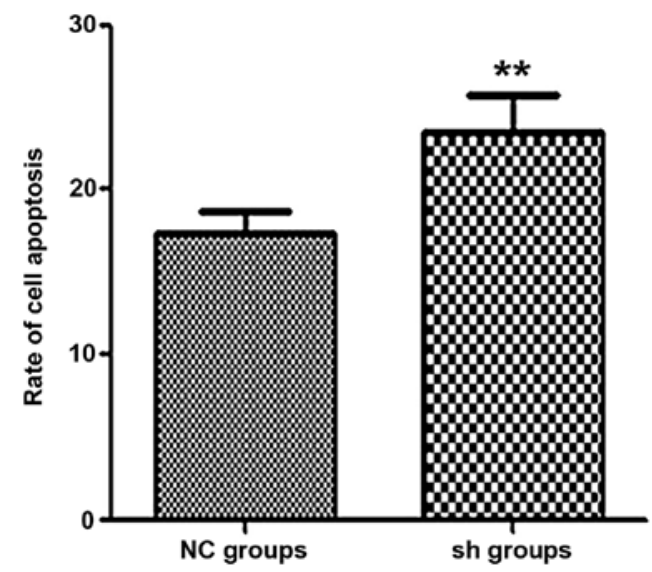

Figure 11. Flow cytomentric analysis of apoptosis. FCM data revealed that the apoptotic rate of MM cells in sh groups was significantly increased compared with NC groups $(* * \mathrm{P}<0.009)$. Experiments were performed in triplicate.

PSMB8 polymorphisms and BTZ resistance, but the direct link remains elusive. Overall, PSMB8 polymorphisms can result in altered proteasome assembly; however, a direct link between PSMB8 polymorphisms and BTZ-resistance remains elusive. Notably, mechanistic studies have demonstrated that overexpression of $\beta 1$ or $\beta 5$ subunits led to increased proteasome activity, and enhanced cell survival following exposure to proteasome inhibitors $(36,37)$. Further studies revealed that in BTZ-resistant RPMI-8226 cells, upregulation of $\beta 5$ expression was clearly opposed by a parallel decrease in $\beta 5 \mathrm{i}$ expression levels, presumably to balance the total proteasome levels in drug-resistant cells compared with parental cells (38). Furthermore, it should be noted that chymotrypsin-like activity probes were used to detect $\beta 5 \mathrm{i}$ and $\beta 1 \mathrm{i}$ subunit activities, which are often downregulated in BTZ-resistant cells, and thus, can influence the overall activity measurements (39). In summary, several studies $(38,40,41)$ have demonstrated that overexpression of $\beta 5$ subunits is an initial cellular response mechanism to BTZ treatment, which may precede the acquisition of mutations following prolonged exposure to BTZ. Better targeted, subunit-specific probes that can discriminate between $\beta 5$, $\beta 5 i$ and $\beta 1$ i-related activities are required to understand these mechanisms in more detail, and may provide an insight into the concentrations of various proteasome inhibitors that lead to the development of drug resistance (39).

It has been previously reported that the direct downregulation of the expression of PSMD10 in MM cells induced an increase in P53 mRNA levels and subsequent upregulation of CDKN1A (p21Waf1/Cip1) and BAX transcripts, which are direct transcriptional targets of P53 (17). PSMD10 acts as an oncoprotein and is involved in the negative regulation of RB1 and P53 tumor suppressors (13). The imbalance of P53 in myeloma cells may play an important role in myeloma pathogenesis, which can be induced through post-transcriptional mechanisms. In our preliminary study, many proteins were identified to be markedly stabilized by BTZ treatment. Similarly, BTZ also stabilized proteasome subunit PSMC1 and proteasome assembly factor PSMD10 (10). Thus, we hypothesize that PSMD10 may be associated with the occurrence and progression of $\mathrm{MM}$, and drug resistance. Thus, a lentivirus-mediated RNAi experiment in MM was designed to validate the growth effects of PSMD10 downregulation on MM cells.

RNAi is a process in which double-stranded RNA is employed to enhance the degradation of endogenous mRNA. siRNA can induce transient and efficient RNAi effects (21). siRNA-producing plasmid vectors allow transient expression of siRNA, and are widely used in gene silencing, but tend to have low transfection efficiency (42). Lentivirus-mediated shRNA systems can produce highly efficient, whole organism expression of shRNAs and allow the induced gene knockdown to be reversed at a given time. This peculiarity of the shRNA system offers specific applications to study gene function in animal experiments, which cannot be achieved with other gene knockdown technologies $(43,44)$. Although conditional lentivirus vectors have been used for several years, the employment of lentiviral vectors expressing shRNA as a therapeutic tool for MM has not been thoroughly explored.

In the present study, lentivirus-mediated shRNA was used to silence endogenous PSMD10 expression and the effects of PSMD10 downregulation on the phenotypes of 
MM RPMI-8226 cells were examined. Three shRNAs were designed to target PSMD10 mRNA and were successfully transfected into 293T cells with the lentivirus plasmids. DNA sequencing and agarose gel electrophoresis further confirmed that three PSMD10 shRNAs were correctly inserted into the multiple cloning sites of the pSIH1-H1-copGFP expression plasmid. Lv-PSMD10-shRNA2 was previously identified as the most effective one, as the $293 \mathrm{~T}$ transfectants exhibited a significantly decreased level of PSMD10 mRNA. In the present study, LvPSMD10-shRNA2 effectively decreased the level of PSMD10 protein, which indicated that our lentivirus-mediated PSMD10-specific shRNA was able to specifically silence the expression of the PSMD10 protein in RPMI-8226 cells. The efficiency of protein silencing was estimated to be $70 \%$ in the present study. However, notably, there was no obvious change in PSMD10 expression at the mRNA level. Therefore, presumably, the knockdown effect on the expression of PSMD10 may take place at the translational level. In addition, the depletion of PSMD10 resulted in increased apoptosis in RPMI-8226 cells in vitro. This effect may be mediated by interference with the P53 signal transduction pathway. The potential oncogenic mechanism of PSMD10 may be to increase the proteasomal degradation of P53 via the MDM2/P53 complex and boost the turnover of P53 in an MDM-dependent manner. In this process, PSMD10 protein binds to MDM2 protein and enhances the ability of MDM2 to ubiquitinate P53 (45). Thus, overexpression of PSMD10 may block P53-dependent apoptosis in human RPMI-8226 MM cells. However, this hypothesis remains to be elucidated in detail. It has been previously demonstrated that when PSMD10 is silenced in hepatocellular carcinoma cell lines with wild-type and mutated P53, a different induced phenotype can be observed in the cells with wild-type P53, with upregulation of P53 levels leading to activation of caspase-9 and the induction of apoptosis (46). BTZ increases P53 protein levels by inhibiting the proteasomal degradation of P53, and subsequently activates the P53 signal transduction pathway, which emphasizes the importance of P53 regulation in the pathogenesis of MM (47). Collectively, the fact that downregulation of PSMD10 expression in myeloma cells induces apoptosis supports the potentially important role of the P53/MDM2 regulatory mechanism in MM.

In the event that PSMD10 shRNA can decrease the resistance of MM to BTZ, it may act as a potential anticancer therapy that could be administered along with BTZ for MM treatment. Therefore, in follow-up experiments, a cell line stably expressing PSMD10 shRNA should be used to review the role of PSMD10 in regulating BTZ sensitivity in MM. Subsequently, different concentrations of BTZ treatment could be used to observe the drug sensitivity in MM cells, and the effect on cell proliferation and apoptosis. In general, the chemosensitivity of MM to BTZ should be examined further, and whether chemosensitivity can be induced or restored by decreasing the expression of PSMD10 should be investigated.

In conclusion, the present study established an efficient method for screening the most effective shRNA the suppression of PSMD10 expression in MM RPMI-8226 cells, and provided a novel strategy for further investigation of the role of PSMD10 in MM. In the exploration of biochemical mechanisms of RNAi pathways, this method is likely to be useful and has the potential to provide more rational strategies for
MM treatment. Collectively, the results of the present study provide further evidence that PSMD10 may have potential as a pharmacological target. The results provide a new perspective for the study of the function of PSMD10 in tumors, and it may be beneficial for future gene therapy strategies in the treatment of MM.

\section{Acknowledgements}

The present study received the support of the National Natural Science Fund of China (no. 81670195). We are sincerely grateful to Professor Tao Zhang and Professor Zi Chen for providing technical assistance and experimental supplies. We are particularly indebted to the help offered by the pharmacological laboratory of the Second Military Medical University and the Central Laboratory of Huashan Hospital.

\section{References}

1. Siegel RL, Miller KD and Jemal A: Cancer statistics, 2015. CA Cancer J Clin 65: 5-29, 2015.

2. National Cancer Institute SEER database: Surveillance, Epidemiology, and End Results Program, 2014 (cited June 30). Available from http://seer.cancer.gov/.

3. Becker N: Epidemiology of multiple myeloma. In: Multiple Myeloma: Recent Results in Cancer Research. Moehler T and Goldschmid H (eds). Springer-Verlag, Berlin, Heidelberg, Germany, pp25-35, 2011.

4. Bergsagel PL: Epidemiology, etiology, and molecularpathogenesis. In: Multiple Myeloma. Richardson PG and Anderson KC (eds). Remedica Publi-shing, London, Chicago, pp1-24, 2004.

5. Liu Y, Xu J, Jiang M, Ni L, Chen Y and Ling Y: Association between functional PSMD10 Rs111638916 variant regulated by MiR-505 and gastric cancer risk in a Chinese population. Cell Physiol Biochem 37: 1010-1017, 2015.

6. Uddin MH, Choi MH, Kim WH, Jang JJ and Hong ST: Involvement of PSMD10, CDK4, and tumor suppressors in development of intrahepatic cholangiocarcinoma of Syrian Golden Hamsters induced by Clonorchis sinensis and N-nitrosodimethylamine. PLoS Negl Trop Dis 9: e0004008, 2015.

7. Li J, Tian F, Li D, Chen J, Jiang P, Zheng S, Li X and Wang S: MiR-605 represses PSMD10/Gankyrin and inhibits intrahepatic cholangiocarcinoma cell progression. FEBS Lett 588: 3491-3500, 2014.

8. Chen BF,Suen YK,Gu S,Li Land Chan WY: A miR-199a/miR-214 self-regulatory network via PSMD10, TP53 and DNMT1 in testicular germ cell tumor. Sci Rep 4: 6413, 2014.

9. Luo T, Fu J, Xu A, Su B, Ren Y, Li N, Zhu J, Zhao X, Dai R, Cao J, et al: PSMD10/gankyrin induces autophagy to promote tumor progression through cytoplasmic interaction with ATG7 and nuclear transactivation of ATG7 expression. Autophagy 12: 1355-1371, 2016

10. Yu T, Tao Y, Yang M, Chen P, Gao X, Zhang Y, Zhang T, Chen Z, Hou J, Zhang Y, et al: Profiling human protein degradome delineates cellular responses to proteasomal inhibition and reveals a feedback mechanism in regulating proteasome homeostasis. Cell Res 24: 1214-1230, 2014.

11. Tomko RJ Jr, Funakoshi M, Schneider K, Wang J and Hochstrasser M: Heterohexameric ring arrangement of the eukaryotic proteasomal ATPases: Implications for proteasome structure and assembly. Mol Cell 38: 393-403, 2010.

12. Higashitsuji $\mathrm{H}$, Itoh $\mathrm{K}$, Nagao $\mathrm{T}$, Dawson $\mathrm{S}$, Nonoguchi $\mathrm{K}$, Kido T, Mayer RJ, Arii S and Fujita J: Reduced stability of retinoblastoma protein by gankyrin, an oncogenic ankyrin-repeat protein overexpressed in hepatomas. Nat Med 6: 96-99, 2000.

13. Higashitsuji H, Higashitsuji H, Itoh K, Sakurai T, Nagao T, Sumitomo Y, Masuda T, Dawson S, Shimada Y, Mayer RJ, et al: The oncoprotein gankyrin binds to MDM2/HDM2, enhancing ubiquitylation and degradation of p53. Cancer Cell 8: 75-87, 2005.

14. Bai Z, Tai Y,Li W, Zhen C, Gu W, Jian Z, Wang Q, Lin JE, Zhao Q, Gong W, et al: Gankyrin activates IL-8 to promote hepatic metastasis of colorectal cancer. Cancer Res 73: 4548-4558, 2013. 
15. Zhen C, Chen L, Zhao Q, Liang B, Gu YX, Bai ZF, Wang K, Xu X, Han QY, Fang DF, et al: Gankyrin promotes breast cancer cell metastasis by regulating Racl activity. Oncogene 32: 3452-3460, 2013.

16. Meng Y, He L, Guo X, Tang S, Zhao X, Du R, Jin J, Bi Q, Li H, Nie Y, et al: Gankyrin promotes the proliferation of human pancreatic cancer. Cancer Lett 297: 9-17, 2010.

17. Misiewicz-Krzeminska I, Sarasquete ME, Quwaider D, Krzeminski P, Ticona FV, Paíno T, Delgado M, Aires A, Ocio EM, García-Sanz R, et al: Restoration of microRNA-214 expression reduces growth of myeloma cells through positive regulation of P53 and inhibition of DNA replication. Haematologica 98: 640-648, 2013.

18. Elbashir SM, Harborth J, Lendeckel W, Yalcin A, Weber K and Tuschl T: Duplexes of 21-nucleotide RNAs mediate RNA interference in cultured mammalian cells. Nature 411: 494-498, 2001.

19. Karagiannis TC and El-Osta A: siRNAs: Mechanism of RNA interference, in vivo and potential clinical applications. Cancer Biol Ther 3: 1069-1074, 2004.

20. Brummelkamp TR, Bernards R and Agami R: A system for stable expression of short interfering RNAs in mammalian cells. Science 296: 550-553, 2002.

21. Paddison PJ, Caudy AA, Bernstein E, Hannon GJ and Conklin DS Short hairpin RNAs (shRNAs) induce sequence-specific silencing in mammalian cells. Genes Dev 16: 948-958, 2002.

22. Reynolds A, Leake D, Boese Q, Scaringe S, Marshall WS and Khvorova A: Rational siRNA design for RNA interference. Nat Biotechnol 22: 326-330, 2004.

23. Barøy T, Sørensen K, Lindeberg MM and Frengen E: shRNA expression constructs designed directly from siRNA oligonucleotide sequences. Mol Biotechnol 45: 116-120, 2010.

24. Kumar SK, Rajkumar SV, Dispenzieri A, Lacy MQ, Hayman SR, Buadi FK, Zeldenrust SR, Dingli D, Russell SJ, Lust JA, et al: Improved survival in multiple myeloma and the impact of novel therapies. Blood 111: 2516-2520, 2008.

25. Rovira M, Rosinol L, Martinez C, Fernández-Avilés F and Blade J: Is there a curative potential of autologous stem cell transplantation in multiple myeloma? Long-term results from a single-centre series. Bone Marrow Transplant 42: S147 (abstract P592), 2009.

26. Gahrton G, Svensson H, Cavo M, Apperly J, Bacigalupo A, Björkstrand B, Bladé J, Cornelissen J, de Laurenzi A, Facon T, et al; European Group for Blood and Marrow Transplantation: Progress in allogenic bone marrow and peripheral blood stem cell transplantation for multiple myeloma: A comparison between transplants performed 1983-93 and 1994-98 at European Group for Blood and Marrow Transplantation centres. Br J Haematol 113: 209-216, 2001.

27. Kyle RA and Rajkumar SV: Multiple myeloma. Blood 111: 2962-2972, 2008.

28. Adams J: The proteasome: A suitable antineoplastic target. Nat Rev Cancer 4: 349-360, 2004.

29. Bianchi G, Oliva L, Cascio P, Pengo N, Fontana F, Cerruti F, Orsi A, Pasqualetto E, Mezghrani A, Calbi V, et al: The proteasome load versus capacity balance determines apoptotic sensitivity of multiple myeloma cells to proteasome inhibition. Blood 113: 3040-3049, 2009.

30. Meister S, Schubert U, Neubert K, Herrmann K, Burger R, Gramatzki M, Hahn S, Schreiber S, Wilhelm S, Herrmann M, et al: Extensive immunoglobulin production sensitizes myeloma cells for proteasome inhibition. Cancer Res 67: 1783-1792, 2007.

31. Ortiz-Navarrete V, Seelig A, Gernold M, Frentzel S, Kloetzel PM and Hämmerling GJ: Subunit of the '20S' proteasome (multicatalytic proteinase) encoded by the major histocompatibility complex. Nature 353: 662-664, 1991.

32. Rivett AJ, Bose S, Brooks P and Broadfoot KI: Regulation of proteasome complexes by gamma-interferon and phosphorylation. Biochimie 83: 363-366, 2001.

33. Niewerth D, Jansen G, Assaraf YG, Zweegman S, Kaspers GJ and Cloos J: Molecular basis of resistance to proteasome inhibitors in hematological malignancies. Drug Resist Updat 18: 18-35, 2015.
34. Agarwal AK,Xing C,DeMartinoGN,MizrachiD,HernandezMD, Sousa AB, Martínez de Villarreal L, dos Santos HG and Garg A: $P S M B 8$ encoding the $\beta 5 \mathrm{i}$ proteasome subunit is mutated in joint contractures, muscle atrophy, microcytic anemia, and panniculitis-induced lipodystrophy syndrome. Am J Hum Genet 87: 866-872, 2010

35. Kincaid EZ, Che JW, York I, Escobar H, Reyes-Vargas E, Delgado JC, Welsh RM, Karow ML, Murphy AJ, Valenzuela DM, et al: Mice completely lacking immunoproteasomes show major changes in antigen presentation. Nat Immunol 13: 129-135, 2011.

36. Chondrogianni N, Stratford FLL, Trougakos IP, Friguet B, Rivett AJ and Gonos ES: Central role of the proteasome in senescence and survival of human fibroblasts: Induction of a senescence-like phenotype upon its inhibition and resistance to stress upon its activation. J Biol Chem 278: 28026-28037, 2003.

37. Chondrogianni N, Tzavelas C, Pemberton AJ, Nezis IP, Rivett AJ and Gonos ES: Overexpression of proteasome beta5 assembled subunit increases the amount of proteasome and confers ameliorated response to oxidative stress and higher survival rates. J Biol Chem 280: 11840-11850, 2005.

38. Franke NE, Niewerth D, Assaraf YG, van Meerloo J, Vojtekova K, van Zantwijk CH, Zweegman S, Chan ET, Kirk CJ, Geerke DP, et al: Impaired bortezomib binding to mutant $\beta 5$ subunit of the proteasome is the underlying basis for bortezomib resistance in leukemia cells. Leukemia 26: 757-768, 2012.

39. Blackburn C, Gigstad KM, Hales P, Garcia K, Jones M, Bruzzese FJ, Barrett C, Liu JX, Soucy TA, Sappal DS, et al: Characterization of a new series of non-covalent proteasome inhibitors with exquisite potency and selectivity for the $20 \mathrm{~S}$ beta5-subunit. Biochem J 430: 461-476, 2010.

40. Rückrich T, Kraus M, Gogel J, Beck A, Ovaa H, Verdoes M, Overkleeft HS, Kalbacher H and Driessen C: Characterization of the ubiquitin-proteasome system in bortezomib-adapted cells. Leukemia 23: 1098-1105, 2009.

41. Niewerth D, Franke NE, Jansen G, Assaraf YG, van Meerloo J, Kirk CJ, Degenhardt J, Anderl J, Schimmer AD, Zweegman S, et al: Higher ratio immune versus constitutive proteasome level as novel indicator of sensitivity of pediatric acute leukemia cells to proteasome inhibitors. Haematologica 98: 1896-1904, 2013b.

42. Heckmann D, Laufs S, Maier P, Zucknick M, Giordano FA, Veldwijk MR, Eckstein V, Wenz F, Zeller WJ, Fruehauf S, et al: A lentiviral CXCR4 overexpression and knockdown model in colorectal cancer cell lines reveals plerixafor-dependent suppression of SDF-1 $\alpha$-induced migration and invasion. Onkologie 34: 502-508, 2011.

43. Christoph T, Bahrenberg G, De Vry J, Englberger W, Erdmann VA, Frech M, Kögel B, Röhl T, Schiene K, Schröder W, et al: Investigation of TRPV1 loss-of-function phenotypes in transgenic shRNA expressing and knockout mice. Mol Cell Neurosci 37: 579-589, 2008.

44. Sacca R, Engle SJ, Qin W, Stock JL and McNeish JD: Genetically engineered mouse models in drug discovery research. Methods Mol Biol 602: 37-54, 2010.

45. Lozano G and Zambetti GP: Gankyrin: An intriguing name for a novel regulator of p53 and RB. Cancer Cell 8: 3-4, 2005.

46. Umemura A, Itoh Y, Itoh K, Yamaguchi K, Nakajima T, Higashitsuji $\mathrm{H}$, Onoue $\mathrm{H}$, Fukumoto $\mathrm{M}$, Okanoue $\mathrm{T}$ and Fujita J: Association of gankyrin protein expression with early clinical stages and insulin-like growth factor-binding protein 5 expression in human hepatocellular carcinoma. Hepatology 47: 493-502, 2008

47. Ooi MG, Hayden PJ, Kotoula V, McMillin DW, Charalambous E, Daskalaki E, Raje NS, Munshi NC, Chauhan D, Hideshima T, et al: Interactions of the $\mathrm{Hdm} 2 / \mathrm{p} 53$ and proteasome pathways may enhance the antitumor activity of bortezomib. Clin Cancer Res 15: 7153-7160, 2009. 